\title{
Effects of gender, cytokine gene polymorphisms and environmental factors on inflammatory responses
}

\author{
Sophia M Moscovis ${ }^{1,2}$, Amanda Cox ${ }^{3}$, Sharron T Hall ${ }^{1,2}$, \\ Christine J Burns ${ }^{1,2}$, Rodney J Scott ${ }^{1,2}$ \\ and C Caroline Blackwell ${ }^{1,2}$
}

\begin{abstract}
Previous studies have indicated that cytokine gene polymorphisms of Indigenous Australians were predominantly associated with strong pro-inflammatory responses. We tested the hypothesis that cells of donors with genetic profiles of inflammatory cytokine single nucleotide polymorphisms (SNPs) similar to Indigenous Australians produce higher proinflammatory responses. PBMCs from 14 donors with genetic profiles for a high risk of strong pro-inflammatory responses and 14 with low-risk profiles were stimulated with endotoxin and effects of gender, IFN- $\gamma$, cigarette smoke extract (CSE) and testosterone on cytokine responses analysed. Cytokines were calculated from standard curves (Luminex 2.3 software). No significant differences were associated with SNP profile alone. Lower pro-inflammatory responses were observed for cells from males with low- or high-risk profiles. For cells from females with high-risk profiles, anti-inflammatory IL-10 responses were significantly reduced. There was no effect of testosterone levels on responses from males. For females, results from IFN- $\gamma$-treated cells showed positive correlations between testosterone levels and IL-I $\beta$ responses to endotoxin for both risk groups and TNF- $\alpha$ for the high-risk group. If interactions observed among CSE, IFN- $\gamma$, genetic background and testosterone reflect those in vivo, these might contribute to increased incidences of hospitalisations for infectious diseases among Indigenous women.
\end{abstract}

\section{Keywords}

Cytokines, gene polymorphisms, cigarette smoke, gender, Indigenous Australians, endotoxin, interferon- $\gamma$

Date received: 26 March 20I4; revised: 16 July 2014; accepted: 20 August 2014

\section{Introduction}

Our previous studies have examined the interactions between genetic background and environmental factors on inflammatory responses to infection. These studies were initiated to explore the apparently higher susceptibility to infection among Indigenous Australians and particularly the severity of these infections. Our findings indicated that among Indigenous Australians their profile of inflammatory cytokine gene single nucleotide polymorphisms (SNPs) might contribute to particularly strong pro-inflammatory responses to infections. ${ }^{1-4}$

A study from central Australia found deaths due to infection to be significantly higher among Indigenous patients. After adjusting for age and year of death, the population-based mortality rate ratios for Indigenous patients was $11.3[95 \%$ confidence interval (CI) 8.0-15.8] and 31.5 (95\% CI 16.1-61.8) for patients $<60$ years of age. Sepsis with Gram-negative enteric organism accounted for half the pathogens involved in these deaths. ${ }^{5}$

Other complex inflammatory-related diseases are also over-represented in the Indigenous Australian population. ${ }^{6}$ Vos et al. identified that ischaemic heart disease $(14 \%)$, diabetes $(12 \%)$, infectious diseases $(9 \%)$ and chronic respiratory diseases $(9 \%)$ were the highest

\footnotetext{
'School of Biomedical Sciences and Pharmacy, Faculty of Health, University of Newcastle, Newcastle, Australia

${ }^{2}$ Hunter Medical Research Institute, Newcastle, Australia

${ }^{3}$ Griffith Health Institute, Molecular Basis of Disease, and School of Medical Science, Griffith University, Southport, Australia

Corresponding author:

Sophia Moscovis, School of Biomedical Sciences and Pharmacy, Faculty of Health, University of Newcastle, PO Box 314, Bondi Junction, NSW 1355, Australia.

Email: s_moscovis@hotmail.com
} 
contributors to the gap between life expectancy of Indigenous compared with non-Indigenous Australians. ${ }^{7}$ The onset and severity of each of these diseases has been linked with alterations in innate immune responses, specifically altered inflammatory responses.

One of the major environmental risk factors for infection is cigarette smoke. There was a significant effect of cigarette smoking on IL-10 responses of individuals with the polymorphism predominant among Indigenous Australians (IL10 G-1082A), a SNP associated with low levels of IL-10 responses. ${ }^{1}$ Other investigators have also previously reported a modifying effect of cigarette smoke on genetic risk for disease. ${ }^{8}$ Given the high prevalence rates of cigarette smoking in Indigenous communities, ${ }^{9}$ consideration of this interaction between genetic and environmental factors could be particularly relevant in understanding their increased risk for disease.

In a recent study, we reported that in a model system pro-inflammatory responses of males were usually lower than those elicited from females. ${ }^{10}$ For females, there were correlations between testosterone levels and some of the pro-inflammatory responses, particularly when IFN- $\gamma$ was used to prime the donor cells prior to stimulation with endotoxin. In this study we applied the model system to compare inflammatory responses elicited from PBMCs from donors with cytokine gene SNP profiles predicted to elicit high pro-inflammatory responses and predominant among Indigenous communities with responses from cells of donors with SNP profiles predominant among non-Indigenous, mainly Caucasian, communities. The studies assessed the interactions between the genetic profiles and the four factors our previous studies indicated could affect responses to the common bacterial endotoxin LPS: gender; IFN- $\gamma$ as a surrogate for virus infection; water-soluble cigarette smoke extract (CSE) as a surrogate for smoking; and testosterone levels, which, in previous studies, had a significant effect on pro-inflammatory responses of female donors. ${ }^{10}$

We tested the hypothesis that donors with the cytokine gene profiles predominant among Indigenous Australians would have significantly higher pro-inflammatory response and/or lower anti-inflammatory responses (IL-10) to LPS stimulation and that their responses might be more significantly affected by factors we had identified in previous studies: IFN- $\gamma$, CSE and testosterone.

\section{Materials and methods}

Buffy coats from 300 donors, aged $20-55 \mathrm{yr}$, were purchased from the Australian Red Cross Blood Service (ARCBS; Sydney, Australia). Ethical permission was obtained from University of Newcastle Human Research Ethics Committee (H-229-0606) and
ARCBS Ethics Committee (07-11NSW-07) for the purchase and use of human buffy coats for the purposes of the study. PBMCs from each donor were collected for the assessment of cytokine gene SNPs. Plasma was stored and assessed for cotinine for evidence of exposure to cigarette smoke, a confounding variable in assessment of cytokine responses. Donors with detectable levels of cotinine were excluded from the analysis. Only ARCBS donor samples that were cleared for infectious agents were received. Buffy coats were processed within $24 \mathrm{~h}$ of collection.

\section{Analysis of exposure to cigarette smoke}

ARCBS donors were assessed for exposure to cigarette smoke by analysing the level of cotinine in the plasma by a semi-quantitative commercial competitive enzyme immunoassay kit according to manufacturer's instructions (OraSure Technologies Inc., Bethlehem, PA, USA). To prevent false negative classification of exposure to cigarette smoke, the qualitative cut-off of the assay was lowered from the recommended $25 \mathrm{ng} / \mathrm{ml}$ to $10 \mathrm{ng} / \mathrm{ml}$. Donors with detectable levels of cotinine were excluded from stimulation assays.

\section{Determination of SNP for donor cells and classification of high and low genetic risk groups}

Six of nine of the polymorphisms assessed in the Indigenous Australian population were used to classify genetic risk for strong pro-inflammatory responses. ${ }^{1-4}$ Three of the nine polymorphisms, ILIRN T $+2018 \mathrm{C}$, IL10 C-592A and IL4 C-589T, although significantly different from the Caucasian population, were excluded from analysis owing to only moderate $(<15 \%)$ differences between allele distribution between Indigenous Australian and Caucasian populations. IL1B C-511T, IL6 G-174C, TNF G-308A, IFNG T + 874A, IL8 A-251T and IL10 G-1082A were assessed by allelic discrimination PCR assays. ${ }^{1-4}$ Alleles predominant in the Indigenous population were predicted to be high genetic risk profiles for strong pro-inflammatory responses; those predominant in the Caucasian population were predicted to be low-risk profiles. SNPs were assigned a dominant mode of penetrance. Donors homozygous for the allele associated with high responses were classified as having double the risk of a donor was heterozygous for that allele. On this basis each donor homozygous for the high risk allele was assigned a value of 2, 1 for heterozygotes and 0 for donors homozygous for the low risk allele. This was carried out for all six SNPs (Table 1) and an overall genetic risk score determined based on the sum of the numerical scores assigned to each of the six SNPs. A panel of 14 donors with high genetic risk profiles (seven males and seven females) and 14 donors with low genetic risk profiles (seven males and seven females) were selected (Table 2). 
Table I. Risk classification for genotype for cytokine SNPs.

\begin{tabular}{lllll}
\hline \multirow{2}{*}{ Gene } & SNP & rs \# & Genotype & $\begin{array}{l}\text { Risk } \\
\text { classification }\end{array}$ \\
\hline ILIB & C-5IIT & rsl6944 & CC & 0 \\
& & & CT & I \\
IL6 & G-I74C & rsI800795 & GG & 2 \\
& & & GC & I \\
& & & CC & 0 \\
TNF & G-308A & rsI800629 & GG & 2 \\
& & & GA & I \\
& & & AA & 0 \\
IFNG & T+874A & rs243056I & TT & 2 \\
& & & TA & I \\
& & & AA & 0 \\
IL8 & A-25IT & rs4073 & AA & 0 \\
& & & AT & I \\
& & & TT & 2 \\
ILI0 & G-1082A & rsI800896 & GG & 0 \\
& & & GA & I \\
& & & AA & 2 \\
& & & &
\end{tabular}

\section{Analysis of testosterone levels}

Total testosterone levels in the plasma of the 28 donors was assessed by Clinical Biochemistry, Hunter Area Pathology Service using the Access immunoassay system (Beckman Coulter, Brea, CA, USA).

\section{Stimulation assay}

PBMCs from the 28 donors were assessed for in vitro cytokine responses to LPS, IFN- $\gamma$ and CSE. The methods for collection, preservation and culture of the PBMCs and preparation of the CSE were described previously. ${ }^{10}$

PBMCs were plated into tissue culture well-plates (Greiner Bio-One, Frickenhausen, Germany) (500 $\mu \mathrm{l} /$ well) at a final concentration of $5 \times 10^{5}$ cells $/ \mathrm{ml}$. Stimulants were immediately added to the cell culture system. Concentrations of stimulants and durations of stimulation were as follows: $24 \mathrm{~h}$ pre-treatment with IFN- $\gamma(10 \mathrm{ng} / \mathrm{ml}) ; 3 \mathrm{~h}$ pre-treatment with $0.001 \mathrm{cig} / \mathrm{ml}$ CSE; $12 \mathrm{~h}$ incubation with LPS $(50 \mathrm{ng} / \mathrm{ml})$.

Cell culture supernatants were collected $12 \mathrm{~h}$ after stimulation with LPS and immediately stored at $20^{\circ} \mathrm{C}$ until assessed for cytokine production. Supernatants were collected by centrifuging cell culture plates at $250 \mathrm{~g}$ for $5 \mathrm{~min}$.

\section{Analysis of cytokine response}

Supernatants were measured for IL-1 $\beta$, IL-6, TNF- $\alpha$, IFN- $\gamma$, IL-8 and IL-10 using Bio-Rad (Hercules,
Table 2. Genetic risk classification of donors.

\begin{tabular}{|c|c|c|c|c|c|c|}
\hline \multirow[b]{2}{*}{ Gender } & \multirow[b]{2}{*}{ Genetic risk } & \multirow[b]{2}{*}{ Age $(y)$} & \multicolumn{3}{|c|}{ Risk tally } & \multirow[b]{2}{*}{ Risk score } \\
\hline & & & 0 & I & 2 & \\
\hline \multirow[t]{14}{*}{ Female } & \multirow{7}{*}{ Low } & 34 & 4 & 0 & 2 & 4 \\
\hline & & 21 & 4 & I & I & 3 \\
\hline & & 55 & 4 & 2 & 0 & 2 \\
\hline & & 35 & 3 & 2 & I & 4 \\
\hline & & 32 & 2 & 4 & 0 & 4 \\
\hline & & 38 & 3 & 2 & I & 4 \\
\hline & & 26 & 4 & I & I & 3 \\
\hline & \multirow[t]{7}{*}{ High } & 37 & I & I & 4 & 9 \\
\hline & & 42 & 0 & 2 & 4 & 10 \\
\hline & & 50 & 0 & 2 & 4 & 10 \\
\hline & & 24 & 0 & 3 & 3 & 9 \\
\hline & & 27 & 0 & 3 & 3 & 9 \\
\hline & & 20 & I & I & 4 & 9 \\
\hline & & 36 & 0 & 3 & 3 & 9 \\
\hline \multirow[t]{14}{*}{ Male } & \multirow[t]{7}{*}{ Low } & 52 & 4 & I & I & 3 \\
\hline & & 31 & 2 & 4 & 0 & 4 \\
\hline & & 36 & 2 & 4 & 0 & 4 \\
\hline & & 54 & 3 & 3 & 0 & 3 \\
\hline & & 33 & 2 & 3 & I & 5 \\
\hline & & 36 & 3 & 2 & I & 4 \\
\hline & & 26 & 4 & 0 & 2 & 4 \\
\hline & \multirow[t]{7}{*}{ High } & 31 & 0 & 3 & 3 & 9 \\
\hline & & 23 & 0 & 2 & 4 & 10 \\
\hline & & 30 & 0 & 3 & 3 & 9 \\
\hline & & 49 & I & I & 4 & 9 \\
\hline & & 21 & 0 & I & 5 & 11 \\
\hline & & 40 & 0 & 3 & 3 & 9 \\
\hline & & 46 & 0 & 3 & 3 & 9 \\
\hline
\end{tabular}

CA, USA) 6-plex assays and the Luminex 200 analyser (Luminex, Austin, TX, USA). Cytokine concentrations $(\mathrm{pg} / \mathrm{ml})$ were calculated from the standard curve using Luminex 2.3 software.

\section{Statistical analysis}

Data were analysed using STATA version 10.0 (Stata Corp., College Station, TX, USA). Mean donor cytokine measurements were used for statistical analysis. The Wilcoxon matched-pairs signed ranks test was used to assess differences in cytokine responses between treatment groups. The significance level was set at $P<0.05$. Mean cytokine responses with SEM are reported. $P$-Values for groups with low cytokine concentrations (i.e. both values $<5 \mathrm{pg} / \mathrm{ml}$ ) were not reported.

Owing to an unexpectedly high variation between experimental wells, sensitivity analysis was performed for groups showing a statistical difference. Within each 
group, samples with high variation (i.e. coefficient of variation $>20 \%$ ) were assessed. For each high-variation sample each measurement used to obtain the mean value was evaluated. The measurement closest to the mean of the comparison group was adopted. In most instances, the adopted value utilised in the sensitivity analysis method did not alter the significant difference between the groups. $P$-Values generated from sensitivity analysis are reported within the text.

The effect of testosterone levels on inflammatory responses was assessed by Pearson's correlation.

\section{Results}

\section{Assignment of genetic risk profiles}

The distributions of SNP genotypes were consistent with Hardy-Weinberg equilibrium. The genotype values assigned for each donor were totalled, providing a total genetic risk score. Seven female and seven male donors with the lowest score were selected for analysis as low-risk donors and seven female and seven male donors with the highest score were selected for analysis as high-risk donors (Table 2). The low-risk group had scores ranging from 2 to 5 and the high risk group scores ranging from 9 to 11 .

\section{Analysis of the experimental groups}

The mean age of low-risk females, high-risk females and high-risk males was $34( \pm 11)$ yr; the mean age of the low-risk male group was $36( \pm 11) \mathrm{yr}$ (Table 2).

\section{Effect of genetic risk profile on cytokine responses}

There were no significant differences in cytokine responses from cells of the 14 donors with the highrisk SNP profile compared with responses from cells of the 14 donors with the low-risk SNP profiles, for any of the conditions tested.

\section{Effect of gender on cytokine responses from cells of donors with the low-risk profile}

Significantly lower cytokine responses were observed when cells from males were pre-treated with IFN- $\gamma$ alone (TNF- $\alpha, P=0.0399)$; IFN- $\gamma$ in combination with LPS (IL-6, $P=0.0181$; TNF- $\alpha, P=0.0350$ ); IFN- $\gamma$ in combination with CSE pre-treatment (IL-6, $P=0.0467$; TNF- $\alpha, P=0.0283$ ); and combination of LPS stimulation following IFN- $\gamma$ and CSE pre-treatment (IL-1 $\beta, P=0.0253$; IL-6, $P=0.0253$; TNF- $\alpha$, $P=0.0181$ ) (Figure 1). Although not statistically significant, there was also a trend for reduced responses in males with a low-risk genotype among all other experimental conditions. When sensitivity analysis of high-variation samples was performed for low-risk donors, five of the seven results remained statistically significant $(P<0.05)$.

For all experimental conditions, there was a trend for reduced responses from cells of males with the lowrisk profile for IFN- $\gamma$, IL-8 and IL-10.

\section{Effect of gender on cytokine responses from cells of donors with the high risk profile}

A significant reduction in IL-6 $(P=0.0405)$, TNF- $\alpha$ $(P=0.0342)$ and IL-8 $(P=0.0455)$ production was observed for cells from male donors pre-treated with CSE. When sensitivity analysis of high-variation samples was performed, the $P$-values were not affected ( $P=0.0405, P=0.0342$ and $P=0.0123$ respectively). A trend for lower IL-8 responses in males was also observed when cells were stimulated with LPS alone $(P=0.0639)$.

IL-10 responses to LPS from cells of male donors were significantly higher $(P=0.0476)$ than cells from females with the high-risk profile. When sensitivity analysis of high-variation samples was performed, the effect on IL-10 was not statistically significant $(P=0.0639)$.

No significant differences in IL-1 $\beta$ and IFN- $\gamma$ responses were associated with gender.

\section{Effect of genetic profile on cytokine responses from cells of female donors}

A significant reduction in cytokine responses was observed in the high risk group when LPS-stimulated cells were pre-treated with IFN- $\gamma$ and CSE (IL- $1 \beta$, $P=0.0350 ; \quad$ IL-6, $P=0.0176 ; \quad$ IL-10, $P=0.0274$ ). When sensitivity analysis of high-variation samples was performed the effect on IL-1 $\beta$ was no longer statistically significant $(P=0.0550)$; the effect on IL-6 and IL-10 remained statistically significant $(P=0.0476$ and $P=0.0274$ respectively).

A significant reduction in IL-10 production was also observed for the high risk group when cells were stimulated with LPS $(P=0.0181)$ and LPS combined with IFN- $\gamma(P=0.0385)$. When sensitivity analysis of high-variation samples was performed, the effect on IL-10 in females remained statistically significant for LPS-stimulated cells $(P=0.0476)$, but not for LPS-stimulated cells pre-treated with IFN- $\gamma$ $(P=0.0610)$ (Figure 2).

There was also a consistent trend for the reduction of IL-10 production in the high-risk group.

The high-risk group also showed a trend for lower TNF- $\alpha$. No trends or significant differences between responses of cells from female donors of the two risk groups were observed for IFN- $\gamma$ of IL-8. 


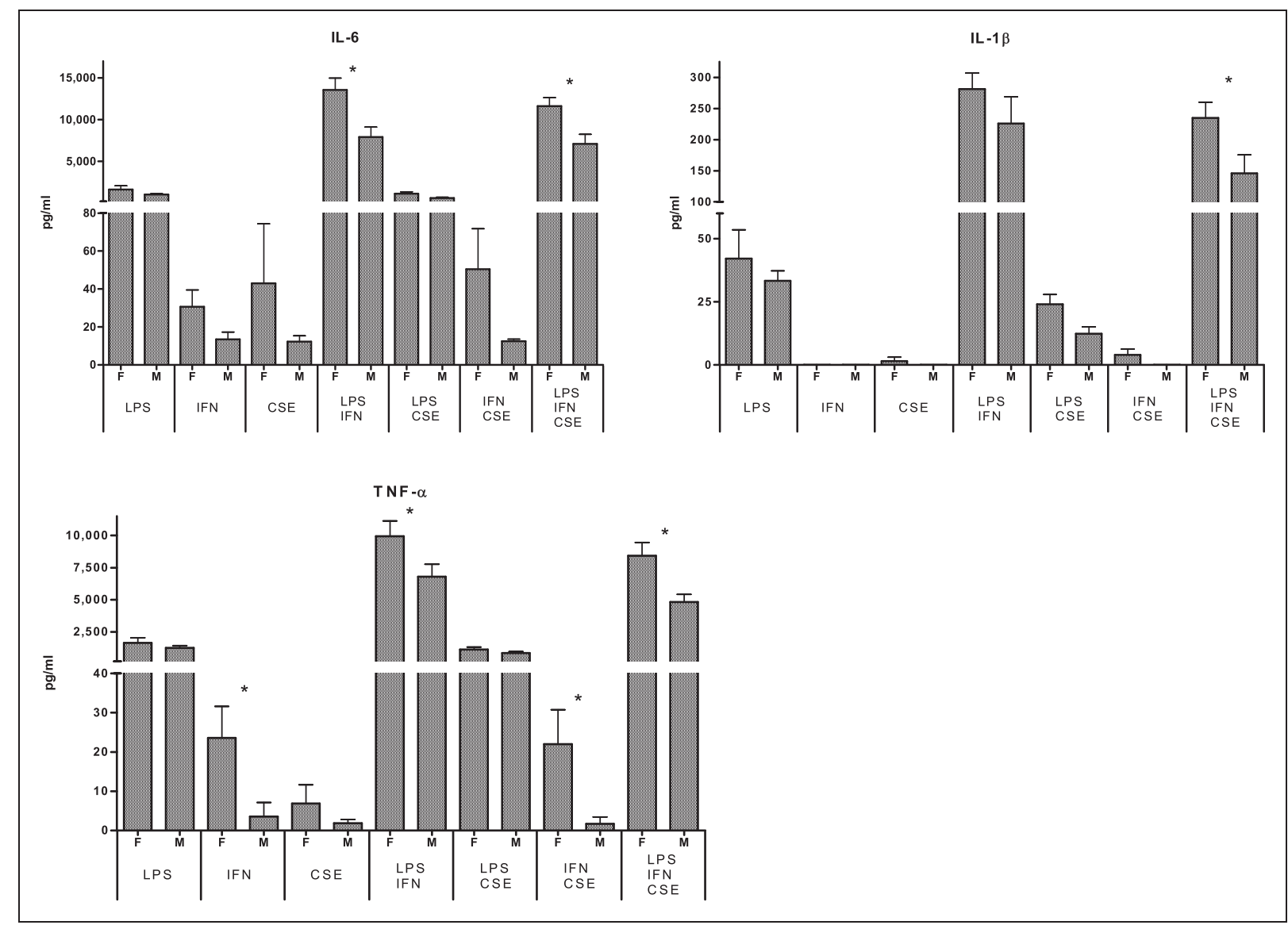

Figure I. Effect of gender on IL-6, IL-I $\beta$ and TNF- $\alpha$ responses: low genetic risk group (seven males, seven females). $P<0.05$.

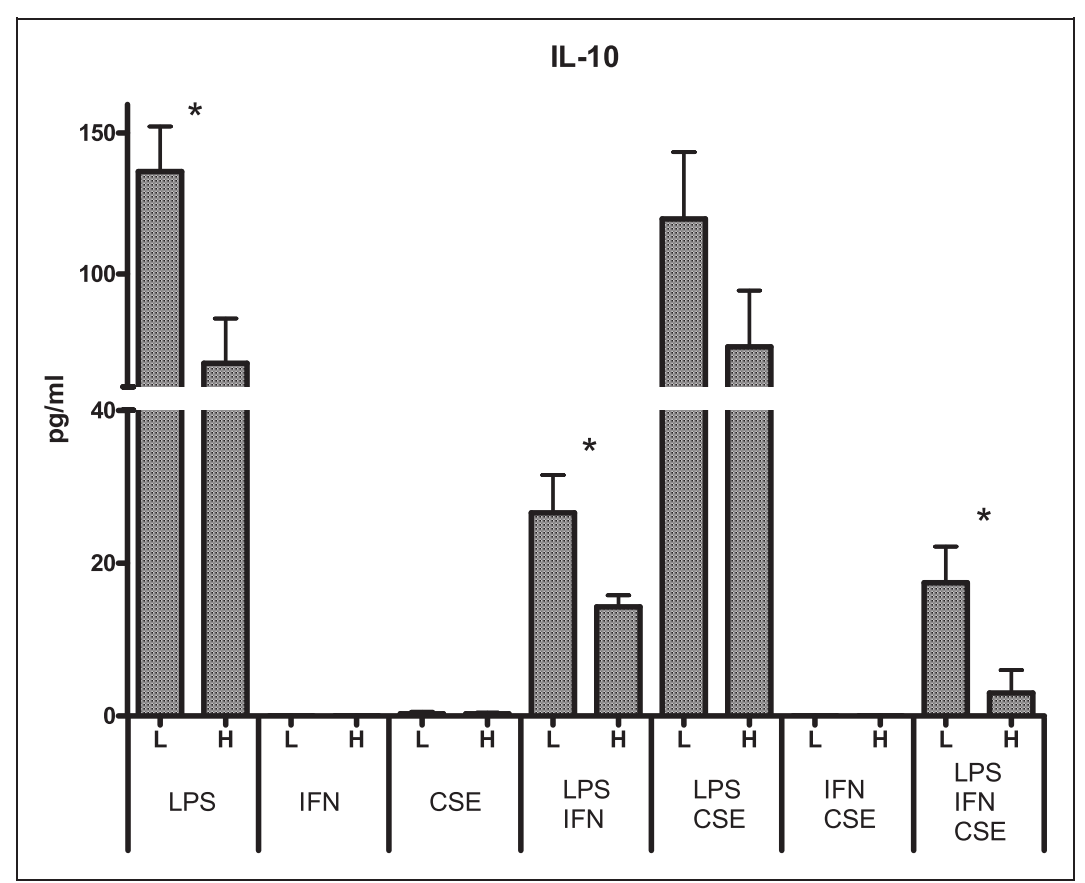

Figure 2. Effect of genetic risk on IL- 10 responses of females [high genetic risk $(H), n=7$; low genetic risk $(L), n=7$ ]. $P<0.05$. 
Effect of genetic profile on cytokine responses from cells of male donors

No significant differences in cytokine responses of cells from males were observed between donors with the high or low risk profiles.

A trend for an increase in cytokine production for the high risk group was observed for IL-1 $\beta$, IL-6 and TNF- $\alpha$ responses to LPS, cells pre-treated with IFN- $\gamma$ alone, and LPS-stimulated cells pre-treated with IFN- $\gamma$ or both IFN- $\gamma$ and CSE. The low risk group showed a trend for lower IFN- $\gamma$ production for all experimental conditions. The high risk group had lower IFN- $\gamma$ when pre-treated with CSE.

\section{Effect of testosterone on inflammatory responses in relation to genetic risk profile}

For any of the experimental conditions tested, there was no correlation between either pro- or anti-inflammatory responses elicited from the cells of male donors and genetic risk profile in relation to testosterone levels $(6.3-21.0 \mathrm{nmol} / 1)$. For cells of female donors, there was a correlation between testosterone levels $(<0.4$ $3.1 \mathrm{nmol} / 1) \mathrm{IL}-1 \beta$, IL-6, IL- 8 and TNF- $\alpha$ responses to LPS; this was observed for the 14 donors for which data for both high risk and low risk profile groups were combined. The effect of testosterone was significant for the combined assessment when IFN- $\gamma$ was present. In the presence of LPS, significant correlations between testosterone levels and IL-1 $\beta$, IL-6 and IL-8 were observed for donors with the high risk profile but only for IL-8 among donors with the low risk profile. When both LPS and IFN- $\gamma$ were present, there was a correlation between IL- $1 \beta$ responses and testosterone levels for donors of both groups. For the high risk group, there was also a correlation between testosterone levels and TNF- $\alpha$ responses. In the presence of LPS and CSE, the only significant correlation noted was between testosterone levels and TNF- $\alpha$ for the high risk group. Although there were significant correlations between testosterone levels and IL-1 $\beta$, IL-6, IL- 8 and TNF- $\alpha$ responses of the 14 donors when all three stimuli were present, the only positive correlation noted for the high or low risk group was between testosterone levels and IL-1 $\beta$ for the high risk group (Table 3).

\section{Discussion}

We tested the hypothesis that the cells of donors with the SNP profiles predicted to elicit strong pro-inflammatory responses would produce higher levels of the pro-inflammatory cytokines and lower levels of the anti-inflammatory IL-10. There was no evidence for this in our results when analysed only in relation to genetic risk profile. Significant differences associated with SNP profile emerged when the analyses assessed the results in relation to sex of the donor.

\section{Analysis of gender on cytokine responses in relation to genetic risk profile}

When assessed in relation to genetic risk group, the reduction of male cytokine responses was evident for

Table 3. Correlations between testosterone levels and inflammatory responses of females in relation to high $(H)$ and low $(L)$ risk cytokine gene profile.

\begin{tabular}{|c|c|c|c|c|c|}
\hline & IL-I $\beta$ & IL-6 & IL-8 & TNF- $\alpha$ & IL-10 \\
\hline \multicolumn{6}{|l|}{ Females } \\
\hline \multicolumn{6}{|l|}{ LPS } \\
\hline$n=14$ & 0.3060 & 0.3030 & 0.4070 & 0.2260 & 0.2200 \\
\hline$L=7$ & 0.3000 & 0.1000 & $* 0.800$ & 0.3000 & -0.5000 \\
\hline $\mathrm{H}=7$ & $* * 0.900$ & $* * 0.900$ & $* * 0.900$ & 0.0100 & 0.4000 \\
\hline \multicolumn{6}{|c|}{$\mathrm{LPS}+\mathrm{IFN}-\gamma$} \\
\hline$n=14$ & $* * * 0.833$ & $* 0.6520$ & 0.1900 & $* * 0.7310$ & 0.4390 \\
\hline$L=7$ & $* * 0.9000$ & 0.5000 & 0.7000 & 0.6000 & 0.3000 \\
\hline $\mathrm{H}=7$ & $* 0.8000$ & 0.7000 & 0.0000 & $* 0.8000$ & 0.0000 \\
\hline \multicolumn{6}{|l|}{$\mathrm{LPS}+\mathrm{CSE}$} \\
\hline$n=14$ & -0.1070 & 0.1630 & 0.1270 & 0.2670 & 0.3920 \\
\hline $\mathrm{L}=7$ & -0.1000 & 0.0000 & 0.3000 & 0.6000 & 0.1000 \\
\hline $\mathrm{H}=7$ & -0.3000 & 0.4000 & 0.2000 & $-0.8000 *$ & 0.6000 \\
\hline \multicolumn{6}{|c|}{$\mathrm{LPS}+\mathrm{CSE}+\mathrm{IFN}-\gamma$} \\
\hline$n=14$ & $* * * 0.8020$ & $* * 0.7110$ & $* * 0.6850$ & $* 0.6180$ & 0.4120 \\
\hline $\mathrm{L}=7$ & 0.7000 & 0.6000 & 0.6000 & 0.7000 & 0.3000 \\
\hline $\mathrm{H}=7$ & $* 0.8000$ & 0.7000 & 0.7000 & 0.1000 & -0.2000 \\
\hline
\end{tabular}

$*=p<0.05, * *=p<0.01$, *** $=p<0.001$. 
both high and low risk groups, for both proinflammatory and anti-inflammatory cytokines. When low-risk donors were assessed, IL-10 responses were lower in males; the opposite trend was observed in a previous study in which low- and high-risk donors were assessed together in relation to sex. ${ }^{10}$

\section{Analysis of genetic risk profile on cytokine responses by gender}

A significant effect of genetic risk was observed for female donors. Females with the SNP profile common among Indigenous Australians had significantly lower levels or showed a trend for lower anti-inflammatory IL-10 for all experimental conditions in which IL-10 was elicited. A similar pattern was observed for IL-6 when all three stimuli (LPS, IFN- $\gamma$, CSE) were present.

The level of IL-10 was lowest when cells from female donors with the SNP profile prevalent among Indigenous Australians were exposed to all three agents. The lowest production of IL-10 was associated with very high levels of pro-inflammatory cytokines and the chemoattractant IL-8. Pro-inflammatory cytokines peaked when cells were treated with bacterial toxin and IFN- $\gamma$; exposure to cigarette smoke only mildly suppressed pro-inflammatory cytokine production.

A genetic predisposition to low IL-10 responses in conjunction with exposure to cigarette smoke, and bacterial and viral infection might result in the greatest risk of tissue damage, severity of infection and susceptibility to auto-immune disease in which the inflammatory responses are dysregulated.

The SNPs over-represented in the Indigenous Australian population were predicted to result in increased pro-inflammatory and decreased anti-inflammatory responses. ${ }^{4}$ An increase in the production of pro-inflammatory cytokines among donors with the Indigenous Australian SNP profile was not observed; however, the reduction of anti-inflammatory responses (IL-10) was observed. The IL10 SNP found overrepresented in the Indigenous Australian population has been previously associated with reduced IL-10 production. ${ }^{1-14}$ A reduction of LPS-induced IL-10 responses in active smokers with the IL10 -1082 allele was observed in a previous study. ${ }^{1}$ In the current study, no significant differences in IL-10 responses were observed when LPS and CSE were assessed in vitro. It was found that when IFN- $\gamma$ was added to the model there was a significant reduction in the level of IL-10 in donors with the IL10 -1082A allele.

The significant reduction of IL-10 production only in high-risk females suggests there could be a gender interaction with the cytokine gene polymorphisms overrepresented in the Indigenous Australian population. A gender interaction has been previously shown for IL-10 responses when assessed in relation to the IL10 $\mathrm{SNP}^{15}$ In contrast to significant correlations noted for pro-inflammatory responses with testosterone levels in female donors (Table 3), there was no effect of testosterone on IL-10 levels.

Review of the hospitalisation rates of Indigenous persons in comparison to rates of other Australians for infectious and parasitic diseases (2005-06) revealed that females had a higher observed/expected ratio than Indigenous males for bacterial diseases (exclusive of intestinal infectious diseases) and pneumococcal septicaemia. ${ }^{6}$ The rate of smoking in Indigenous Australians is approximately $50 \%$. The interactions observed with CSE in relation to cytokine responses in the females in this study might contribute to the increased incidence of hospitalisations for infectious diseases observed in Indigenous women.

A trend was observed in male donors with the SNP profile prevalent among Indigenous Australians, which was opposite to the effect observed in females; an increase in pro-inflammatory and anti-inflammatory cytokines was observed. This has not been reported previously, and overall rates of infectious diseases are increased in Indigenous males compared with nonIndigenous Australian counterparts. Further investigation is required to determine if the cytokine responses associated with the Indigenous Australian SNP profile are significantly affected by gender.

\section{Study limitations}

This study was based on the minimum number of samples to detect a $10 \%$ difference in cytokine response with $80 \%$ power $(\alpha=0.05)$. Increasing the sample size for each group was limited by financial constraints and the number of samples available for testing. The donors were from a predominantly Caucasian population as reflected in the distribution of the SNP examined. The studies need to be repeated with cells from Indigenous donors.

\section{Conclusions}

The study findings indicate that: (1) cytokine gene polymorphisms overrepresented in Indigenous Australians were associated with reduced anti-inflammatory IL-10 responses elicited by LPS from cells of female donors; and (2) in females, the effect of exposure to cigarette smoke, bacterial and viral infection and SNP overrepresented in the Indigenous Australian population yielded the lowest anti-inflammatory IL-10 response in parallel with sustained high pro-inflammatory responses.

\section{Funding}

The work was funded by the Foundation for the Study of Infant Death (FSID) (UK), Babes in Arms (UK), Hunter Medical Research Institute (HMRI), the University of Newcastle and the John Hunter Children's Research Foundation. 


\section{Conflicts of interest}

The authors do not have any potential conflicts of interest to declare.

\section{References}

1. Moscovis SM, Gordon AE, Al Madani OM, et al. Interleukin-10 and sudden infant death syndrome. FEMS Immunol Med Microbiol 2004; 42: 130-138.

2. Moscovis SM, Gordon AE, Al Madani OM, et al. IL6 G-174C associated with sudden infant death syndrome in a Caucasian Australian cohort. Hum Immunol 2006; 67: 819-825.

3. Moscovis SM, Gordon AE, Al Madani OM, et al. Interleukin 1-beta responses to bacterial toxins and sudden infant death syndrome. FEMS Immunol Med Microbiol 2004; 42: 139-145.

4. Cox AJ, Moscovis SM, Blackwell CC, et al. Cytokine polymorphisms in Indigenous Australians. Innate Immun 2014; 20: 431-439.

5. Einsiedel LJ, Fernandes LA and Woodman RJ. Racial disparities in infection-related mortality at Alice Springs Hospital, Central Australia, 2000-2005. Med J Austral 2008; 188: 568-571.

6. Australian Bureau of Statistics \& Australian Institute of Health and Welfare. The health and welfare of Australia's Aboriginal and Torres Strait Islander Peoples. Canberra: ABS \& AIHW, 2008.

7. Vos T, Barker B, Begg S, et al. Burden of disease and injury in Aboriginal and Torres Strait Islander Peoples: the Indigenous health gap. Int J Epidemiol 2009; 38: 470-477.
8. Ruddock ME, Cox AJ, Ziegler JT, et al. Cigarette smoking status has a modifying effect on the association between polymorphisms in KALRN and measures of cardiovascular risk in the diabetes heart study. Genes Genomics 2011; 33: 479-486.

9. Australian Institute of Health and Welfare. The health and welfare of Australia's Aboriginal and Torres Strait Islander people, an overview 2011. Canberra: AIHW, 2011.

10. Moscovis SM, Hall ST, Burns CJ, et al. The male excess in sudden infant death. Innate Immun 2014; 20: 24-29.

11. Stanislova SA, Miteva LD, Karakolev ZT, et al. Interleukin10-1082 promoter polymorphism in association with cytokine production and sepsis susceptibility. Intensive Care Med 2006; 32: 260-266.

12. Miteva L and Stanislova S. The combined effect of interleukin (IL)-10 and IL-12 polymorphisms on induced cytokine production. Hum Immunol 2008; 69: 562-566.

13. Turner DM, Williams DM, Sankaran D, et al. An investigation of polymorphism in the interleukin-10 gene promoter. Eur $J$ Immunogenet 1997; 24: 1-8.

14. Crawley E, Kay R, Sillibourne J, et al. Polymorphic haplotypes of the interleukin-10 5' flanking region determine variable interleukin-10 transcription and are associated with particular phenotypes of juvenile rheumatoid arthritis. Arthritis Rheum 1999; 42: 1101-1108.

15. Tedde A, Laura Putignano A, Bagnoli S, et al. Interleukin-10 promoter polymorphisms influence susceptibility to ulcerative colitis in a gender-specific manner. Scand J Gastroenterol 2008; 43: $712-718$. 